DOI: http://doi.org/10.21698/simi.2017.0032

\title{
ENVIRONMENTAL FACTORS - POTENTIAL RESERVOIRS OF NOSOCOMIAL INFECTIONS
}

\author{
Alina Roxana Banciu, Mihai Nita-Lazar, Liliana Daniela Niculescu \\ National Reasearch and Development Institute for Industrial Ecology - ECOIND, 71-73 Drumul \\ Podu Dambovitei, 060652 Bucharest, Romania, alina.banciu@incdecoind.ro
}

\begin{abstract}
All infections follow the basic epidemiological pattern that if recognized early helps to apply the measures to prevent and control pathologies. The epidemiological process cannot be triggered in the absence of the microorganism's reservoir and therefore it is very important to control the potential reservoirs involved in nosocomial infections. The etiological structure of nosocomial infections varies and includes a wide variety of potentially or strictly pathogenic bacteria that have native or acquired proprieties of virulence and antibiotic resistance. The aim of this project was to monitor the potential role of the environmental risk factors involved in outbreak nosocomial infections. The microbiological studies from an emergency hospital from Bucharest have been started in January 2016 and samples were quarterly collected from drilling and tap water, waste water, air and surfaces. There were performed quantitative analyzes of microbiological contamination level and qualitative analyzes for pathogenic and resistant bacteria detection. Some pathogenic bacteria have been identified in the waste water and drilling water, indicating their interference. The antibiotic resistance properties were observed especially in bacteria from waste waters. Bacteria from vegetables from food preparation were detected in air samples and surfaces tests from kitchen. The results indicated that environmental factors from a medical unit could constitute a reservoir of microorganisms that can trigger nosocomial infections.
\end{abstract}

Keywords: antibiotic resistance, infection pathogenic bacteria

\section{Introduction}

Nosocomial infections, also called "hospital acquired infections" are infections acquired during hospitalization which are not present or incubating at administration. Studies throughout the World Health Organization (WHO) documents showed that nosocomial infections have been a major cause of morbidity and mortality.

Contact between patients and a microorganism during hospitalization does not necessarily result in the development of clinical disease. Other factors influence the nature of nosocomial infections such as antibiotic resistance of microorganisms, intrinsic virulence, infected materials and hospital environmental conditions. Water, air and surfaces from hospital must meet aseptic conditions. Drinking water should be safe for ingestion. Fecal contamination most likely is sufficient to cause infections during food preparation, washing or in aerosols, unless adequate treatment is provided (WHO 2002).

Nosocomial waterborne pathogens may reach patients through several modes of transmission. Colonization of healthcare waterworks facility could occur in the proximal infrastructure, in the distal water outlets, or both. Hospital should have 


\section{INTERNATIONAL SYMPOSIUM "THE ENVIRONMENT AND THE INDUSTRY", SIMI 2017, PROCEEDINGS BOOK}

prospective water safety plants that include preventive measures, as prevention is preferable to remediation of the contamination from hospital water distribution systems (Decker et al. 2014).

Biofilms develop on all surfaces in contact with non-sterile water. Pathogens, even present below detection limit in water, could attach to biofilms generating a pathogenic environmental reservoir, a potential source of water contamination. Particularly critical are the water systems of hospital where biofilm borne pathogens can considerably contribute to water-associated nosocomial infections. Biofilm can represent the source of pathogens at continuous exposure of patients, care-givers and all surfaces which may come into contact with contaminated water (Wingender et al. 2011). Many outbreaks of waterborne nosocomial infections have been reported and an estimated 1400 deaths occur each year in the United States as a result of waterborne nosocomial pneumonias caused by Pseudomonas aeruginosa (Anaissie et al. 2002). In addition, other hygienically relevant bacteria such as Escherichia coli, Klebsiella oxytoca were detected in drinking water from hospitals

Inanimate surfaces have often been described as a source for outbreaks of nosocomial infections. The most common nosocomial pathogens may well survive or persist on surfaces for months and can thereby be a continuous source of transmission if no regular preventive surface disinfection is performed (Kramer et al. 2006).

Health centers constantly generate wastewater and a consequent discharge of effluents that require appropriate treatment and disinfection. Hospital wastewater releases a variety of substances such as pharmaceuticals, disinfectants, antimicrobial drugs and multiresistant bacteria (Emmanuel et al. 2005; (Emmanuel et al. 2009). Beta-lactam antibiotics are commonly used to treat hospitalized patients infected by Gram negative bacteria. The production of beta-lactamases is the principal mechanism involved in the inactivation of these antibiotics and the ESBLs (extended-spectrum betalactamases) confer resistance to the penicillin, third-generation of cephalosporins and aztreonam. These resistant microorganisms get into the hospital wastewater, for instance the pathogenic and antibiotic resistant bacteria detected in the wastewater treatment plant of hospital from Rio de Janeiro, Brazil, indicated a potential risk for the microbiological pollution of water resources and a way of transmitting resistant bacteria (Chagas et al. 2011).

Over all, studies conducted during 2012 on more than 1000 hospitals in more than 30 countries allowed European Center for Disease Prevention and Control to estimate that every day in European hospitals around 8000 patients suffer from at least one nosocomial infection. Acquired pathology is an important public health issue since in 2013, 366 cases of nosocomial infection were recorded in Romania (ECDC 2013).

As regards the control of nosocomial infections, the knowledge of the degree of water, air, objects and surfaces contamination with potential pathogenic microorganisms, constitutes an important criterion for the assessment by hygiene conditions.

The aim of this project was to monitor the environmental risk factors potentially involved in outbreak nosocomial infections in an emergency unit from Bucharest, Romania.

\section{Materials and Methods}

The experimental study has been started in the first quarter of 2016 then quarterly were collected samples from the hospital drillings, dispensers and municipal water supply, wastewater plant, air and surfaces (ISO 19458 2006). 


\section{INTERNATIONAL SYMPOSIUM "THE ENVIRONMENT AND THE INDUSTRY", SIMI 2017, PROCEEDINGS BOOK}

The quantitative analyses of drinking water indicators (L 458 2002) based on colony count technique on yeast extract agar (Oxoid, UK) for heterotrophyc bacteria (ISO 6222 1999) and membrane filtration method for coliform bacteria and Enterococcus spp. The Chromogenic coliform agar (Biolife, It.) was used to isolate and count coliform bacteria and E. coli (ISO 9308-1 2014) Slanetz \& Bartley agar (Biolife, It.) and Bile aesculine agar (Biolife, It.) were used for detection of Enterococcus (ISO 7899-2 2000)

The most probable number technique on Colilert-18 (IDEXX, UK) was performed for detect coliform bacteria from wastewater samples (ISO 9308-2 2012). Enterococcus spp was detected through the same membrane filtration method as for the drinking water samples.

Aeromicroflora samples were taken by the Koch method and the surfaces samples were taken with sterile strips. The sampling points have been established according to risk activities such as kitchen and pediatrics.

The qualitative analyses identified the bacterial strains based on metabolic reactions by the Omnilog system (Biolog, USA) and detected the antibiotic resistance profile (CLSI 2015).

\section{Results and Discussion}

There were performed quantitative analyses for the microbiological indicators of water samples and quantitative analyses for identification of potentially pathogenic and resistant bacteria from the collected samples.

The results of microbiological analyses of drinking water from 2016 sampling campaign indicated a high degree of contamination with potentially pathogenic bacteria in drillings water and dispenser's water from pediatrics (Banciu et al. 2016). Based on these previously reported results the medical unit decided to renounce of the use of own drillings water and dispensers. The microbiological analyses for drinking water were performed during 2017 only from the municipal water supply. The values of microbiological indicators analyzed in the first two quarters of 2017 for drinking water were within the limits imposed by the present legislation.

The quantitative results of microbiological indicators of wastewater indicated a significantly increased values of Gram negative bacteria (Figure 1) among them we identified Raoutella ornitholityca, Proteus mirabilis, Klebsiella oxytoca, Serratia liquefaciens. These microorganisms presented resistance to beta-lactams, the most commonly used substances in treatment procedures from the studied hospital.

The air and sanitation tests of the kitchen tables resulted in the identification of bacteria with food origin, Brevibacterium linens, Rhizobium vitis and Paenibacillus provencensis.

The sanitation test of the water tap from the hospital kitchen detected Rahnella aquatillis, resistant to the majority of beta-lactam antibiotics (Figure 2) and Klebsiella oxytoca resistant to Gentamicin and Chloramphenicol (Figure 3). 


\section{INTERNATIONAL SYMPOSIUM "THE ENVIRONMENT AND THE INDUSTRY", SIMI 2017, PROCEEDINGS BOOK}
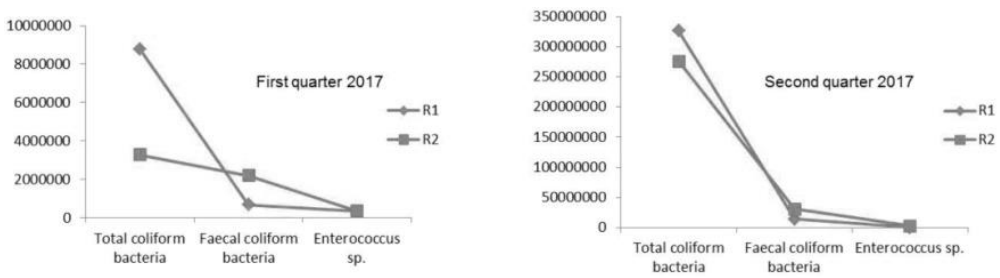

Figure 1. Density of microbiological indicators in wastewater samples for an emergency hospital (Bucharest, Romania) in the first two quarters of 2017

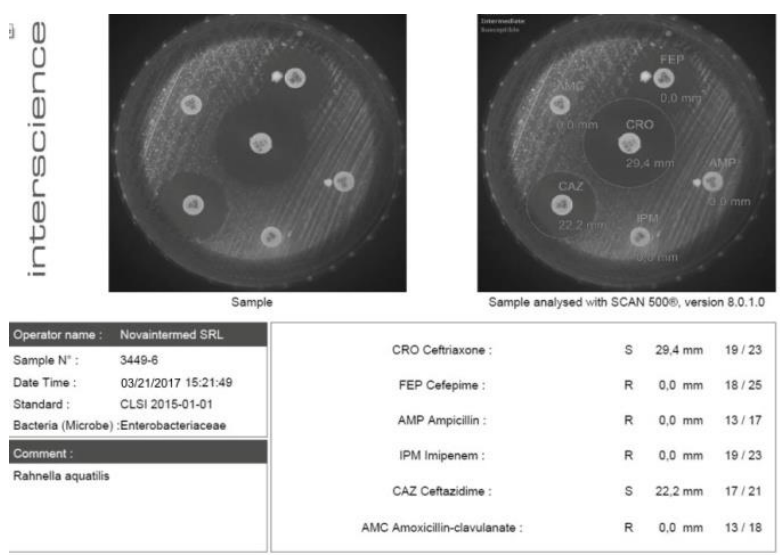

Figure 2. Antibiotic susceptibility testing for Rahnella aquatilis isolated from sanitation test of water tap from kitchen - emergency hospital from Bucharest, Romania.

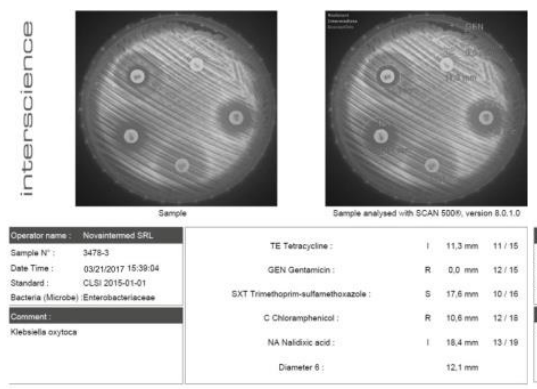

(a)

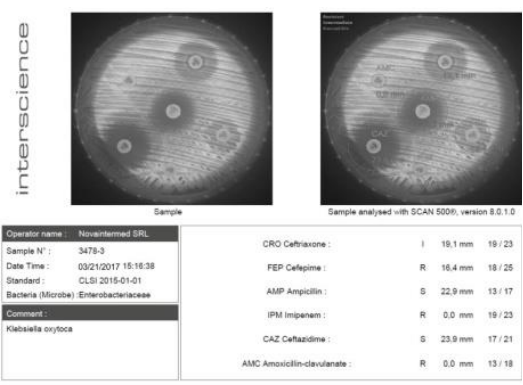

(b)

Figure 3. a. Beta-lactams susceptibility testing for Klebsiella oxytoca isolated from sanitation test of water tap from the kitchen - emergency hospital from Bucharest, Romania; b. General antibiotic susceptibility testing for Klebsiella oxytoca isolated from sanitation test of water tap from kitchen - emergency hospital from Bucharest, Romania. 


\section{INTERNATIONAL SYMPOSIUM "THE ENVIRONMENT AND THE INDUSTRY", SIMI 2017, PROCEEDINGS BOOK}

The water on this tap was provided by the city network and it was assumed that the water met the highest microbiological quality and the quantitative results of this study demonstrated it. It could be speculated that the contamination with antibiotic resistant bacteria came entirely from the hospital environment.

The results of surfaces analyses in the pediatric unit indicated the presence of Pseudomonas aeruginosa resistant to Cefepime, Aztreonam and Ceftazidime (Figure 4).

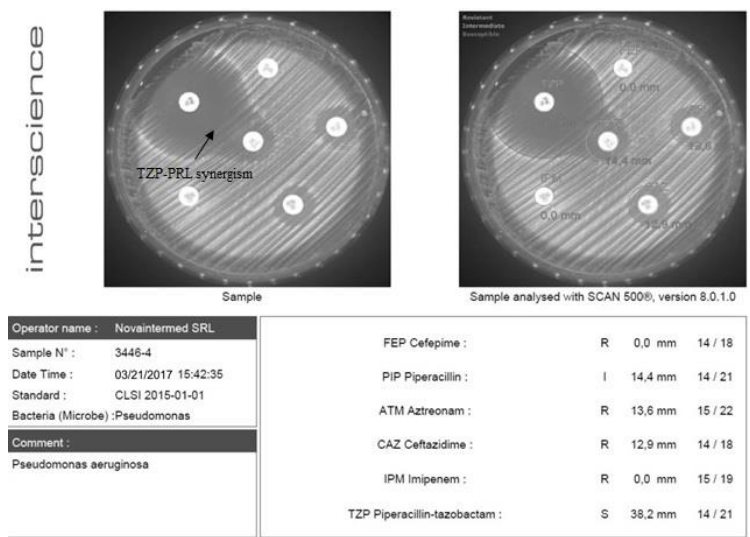

Figure 4. Antibiotic susceptibility testing for Pseudomonas aeruginosa isolated from sanitation test of pediatric unit - emergency hospital from Bucharest, Romania

The expression of beta-lactam activity and the piperacillin-tazobactam susceptibility of the Pseudomonas aeruginosa showed antibiotic resistance profile (Figure 4). The aeromicroflora from the pediatric room showed the presence of Enterobacter aerogenes resistant to beta-lactams and other some antibiotics (Figure 5).

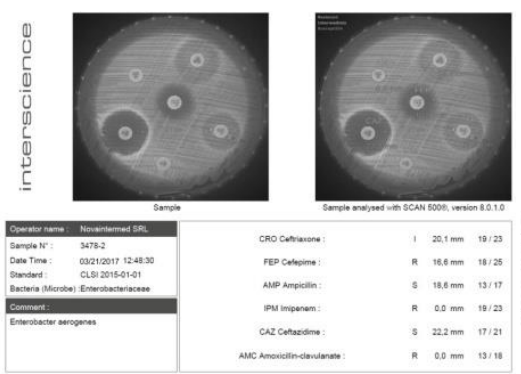

(a)

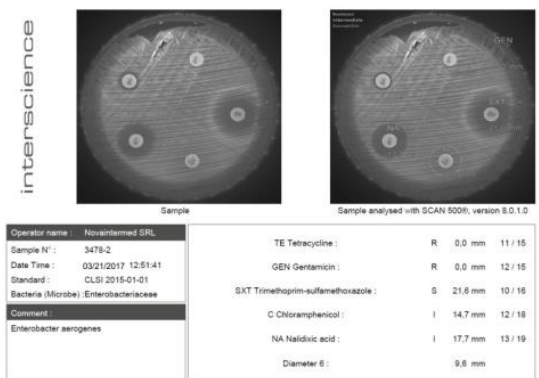

(b)

Figure 5. a. Beta-lactams susceptibility testing for Enterobacter aerogenes provided from aeromicroflora of pediatric room - emergency hospital from Bucharest, Romania; b. General antibiotic susceptibility testing for Enterobacter aerogenes provided from aeromicroflora of pediatric room - emergency hospital from Bucharest, Romania. 


\section{INTERNATIONAL SYMPOSIUM "THE ENVIRONMENT AND THE INDUSTRY", SIMI 2017, PROCEEDINGS BOOK}

Resistance mechanisms associated with modification of membrane permeability such as porin alteration and active efflux are released of Enterobacter aerogenes environmental matrices of hospital.

\section{Conclusions}

The results of the microbiological indicators for municipal water supply showed that this water was fine for drinking purposes.

The wastewater analyses indicated a high density of fermentative bacteria and the necessity of a more efficient treatment plant could be taken into consideration.

The microbiological experiment for hygiene conditions highlighted that bacteria might be involved in the outbreak nosocomial infections. Some of these bacteria had a food preparation origin and could trigger pathologies at immunocompromised patients due to its antibiotic resistance properties. Pseudomonas aeruginosa and Enterobacter aerogenes were two species of considerable medical importance due to their multidrug resistant properties.

The experimental study from 2016 to the present has led to the decommissioning of water from own drillings and automatic dispensers. Correlation between beta-lactams commonly used in hospital and antibiotics those for which microorganisms showed resistance may suggest more attention to controlling the antimicrobials administration.

The data obtained demonstrated that environmental factors of hospitals, especially disinfection conditions and presence of antibiotic resistant microorganisms, may represent reservoirs for nosocomial infections.

The adoption of increased prevention and control measures is required to eliminate the occurrence of risk factors. The monitoring of the sterilization and disinfection conditions in the various sections of hospital is part of the international program of surveillance and control of nosocomial infections.

\section{Acknowledgements}

CORE Program PN 16250105 - Investigation of the pathogenic profile and antibiotic resistance of microorganisms potentially involved in causing nosocomial infections.

\section{References}

Anaissie, EJ, Penzak, SR \& Dignani, MC 2002, 'The hospital water supply as a source of nosocomial infections - A plea for action', Archives of Internal Medicine, vol. 162, no. 13, pp. 1483-1492.

Banciu, A, Niculescu, LD, Nita-Lazar, M \& Lucaciu, I 2016, 'Antibiotics implications on the outbreak nosocomial infections', International Symposium "The Environment and the Industry" SIMI 2016, National Research and Development Institute for Industrial Ecology ECOIND, Bucharest, pp. 105111.

Chagas, TPG, Seki, LM, Cury, JC, Oliviera, JAL, Davila, AMR, Silva, DM \& Asensi MD 2011, 'Multiresistance, beta-lactamase-encoding genes and bacterial diversity in hospital wastewater in Rio de Janeiro, Brazil', Journal of Applied Microbiology, vol. 111, no. 3, pp. 572-581.

CLSI 2015, Clinical and Laboratory Standards Institute, Performance standard for Antimicrobial Susceptibility Testing, M100S, 26 ${ }^{\text {th }}$ Edition. 


\section{INTERNATIONAL SYMPOSIUM "THE ENVIRONMENT AND THE INDUSTRY", SIMI 2017, PROCEEDINGS BOOK}

Decker, BK \& Palmore, TN 2014, 'Hospital water and opportunities for infection prevention', Current Infectious Disease Reports, vol. 16, no. 432, pp. 1-8.

ECDC 2013, European Center for Disease Prevention and Control, 'Each day, one in 18 patients in European hospitals has a healthcare-associated infection: ECDC estimates', Press Release, ECDC, Stockholm, pp.1-2.

Emmanuel, E, Pierre, MG \& Perrodin, Y 2009, 'Groundwater contamination by microbiological and chemical substances released from hospital wastewater: Health risk assessment for drinking water consumers', Environment International, vol. 35, no. 4, pp. 718-726.

Emmanuel, E, Perrodin, Y, Keck, G, Blanchard, JM \& Vermande, P 2005 , 'Ecotoxicological risk assessment of hospital wastewater: a propose framework for raw effluents discharging into urban sewer network', Journal of Hazardous Materials, vol. 117, no. 1, pp. 1-11.

ISO 19458 2006, 'Water quality. Sampling for microbiological analyses, International Organization for Standardization.

ISO 6222 1999, 'Water quality. Enumeration of culturable micro-organisms - colony count by incubation in a nutrient agar culture medium', International Organization for Standardization.

ISO 9308-1 2014, 'Water quality. Enumeration of Escherichia coli and coliform bacteria. Part 1. Membrane filtration method for waters with low bacterial background flora', International Organization for Standardization.

ISO 7899-2 2000, 'Water quality. Detection and enumeration of intestinal enterococci. Part 2. Membrane filtration method', International Organization for Standardization.

ISO 9308-2 2012, 'Water quality. Enumeration of Escherichia coli and coliform bacteria. Part 2. Most probable number method', International Organization for Standardization.

Kramer, A, Schwebke, I \& Kampf, G 2006, 'How long do nosocomial pathogens persist on inanimate surface? A systematic review', BMC Infections Diseases, vol. 6 , no. 130 , pp. 1-8.

Law 458 2002, (r1) the drinking water quality law, Romanian Official Monitor from 28 August 2002.

Wingender, J \& Flemming, HC 2011, 'Biofilms in drinking water and their role as reservoir for pathogens', Journal of Hygiene and Environmental Health, vol. 214, pp. 417-423.

WHO 2002, Department of Communicable disease, Surveillance and Response, Prevention of hospitals - acquired infections, A practical guide, $2^{\text {nd }}$ edition, World Health Organization. 